\title{
Rendimiento de forraje de tres genotipos de Lotus corniculatus L., en función de la edad al corte
}

\section{Forage yield of three genotypes of Lotus corniculatus L., according to age at cut}

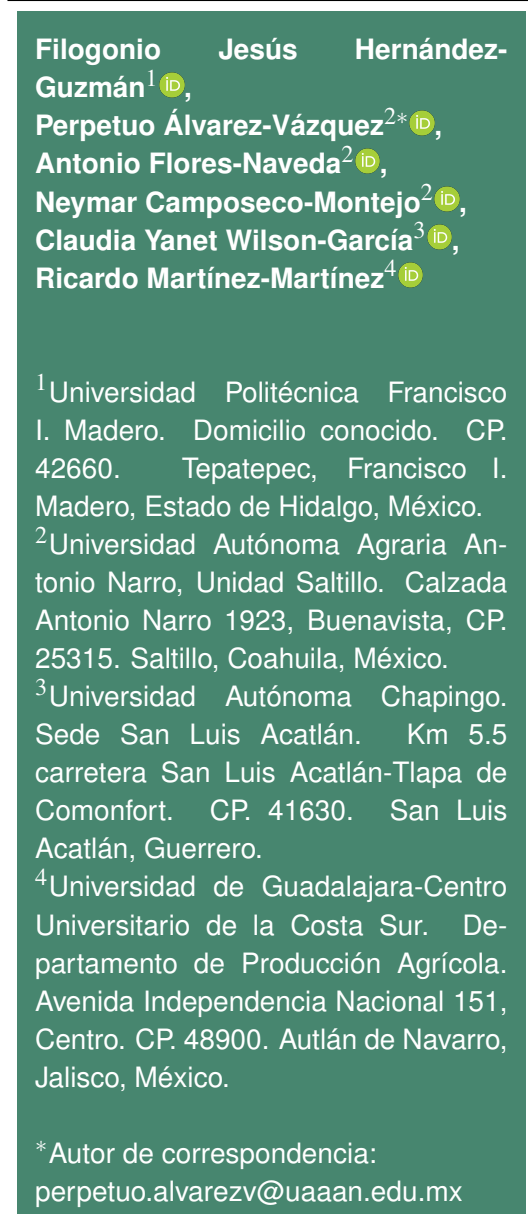

Nota científica

Recibida: 02 de marzo 2021 Aceptada: 23 de septiembre 2021

Como citar: Hernández-Guzmán FJ, Álvarez-Vázquez P, Flores-Naveda A, Camposeco-Montejo N, WilsonGarcía CY, Martínez-Martínez R (2021) Rendimiento de forraje de tres genotipos de Lotus corniculatus L., en función de la edad al corte. Ecosistemas y Recursos Agropecuarios Núm. Esp. II: e2937. DOI: 10.19136/era.a8nll.2937
RESUMEN. El objetivo fue determinar el efecto del momento del corte sobre la respuesta productiva de tres genotipos de L. corniculatus. El diseño fue completamente al azar, con tres repeticiones. El mayor y menor rendimiento se presentó a los 35 y 7 DDR con 5.48 y $0.72 \mathrm{~g} \mathrm{MS} \mathrm{planta}^{-1}$. La hoja fue la que más aportó al rendimiento. La mayor y menor relación hoja:tallo se registró a los 7 (5.71) y 42 DDR (2.39), siendo el genotipo 255301 superior al resto $(p>0.05)$. La altura y el peso del tallo se incrementaron de los 7 y 49 DDR. En conclusión, la edad de cosecha afectó a los genotipos de $L$. corniculatus, siendo mayor el 255301 al resto de los genotipos en la altura, relación hoja:tallo, y peso por tallo individual, no en rendimiento de forraje y composición botánica y morfológica.

Palabras clave: Comportamiento productivo, composición botánicamorfológica, Lotus corniculatus, rendimiento de forraje.

ABSTRACT. The objective was to determine the effect of the cutting moment on the productive response of three genotypes of $L$. corniculatus. The design was completely randomized, with three replications. The highest and lowest yields were presented at 35 and 7 DDR with 5.48 and $0.72 \mathrm{~g} \mathrm{DM}$ plant $^{-1}$. The blade was the one that contributed the most to the performance. The highest and lowest leaf:stem ratio was recorded at 7 (5.71) and 42 RDD (2.39), with the 255301 genotype being superior to the rest $(p>0.05)$. The height and weight of the stem increased from 7 and 49 RDD. In conclusion, the harvest age affected the $L$. corniculatus genotypes, with 255301 being greater than the rest of the genotypes in height, leaf: stem ratio, and weight per individual stem, not in forage yield and botanical and morphological composition.

Key words: Productive performance, botanical-morphological composition, Lotus corniculatus, forage yield. 


\section{INTRODUCCIÓN}

Lotus corniculatus L., es reconocida como una especies forrajera con un alto potencial productivo (Naydenova et al. 2015). Por su alto valor nutricional y formas de utilización (García et al. 2014), ha sido comparada con la alfalfa (Medicago sativa L.) y trébol blanco (Trifolium repens L.) (Escaray et al. 2012). Se adapta a diferentes condiciones edáfoclimáticas; deficiencia de fósforo, altas concentraciones de aluminio, magnesio, bajas temperatura y sequía (Vasileva et al. 2014). Si embargo, como en la mayoría de las especies forrajeras, la productividad y persistencia de L. corniculatus, son determinadas por el manejo del cultivo; intensidad y frecuencia de defoliación (Da Silva et al. 2020). Por tanto, el análisis de crecimiento de una especie forrajera se enfoca en describir el comportamiento de sus componentes morfológicos, que son influenciados por la luz, la temperatura, la disponibilidad de agua y por manejos de la defoliación (Sousa et al. 2010). Bajo este enfoque, se han evaluado variables productivas como la altura de planta, índice de área foliar y porcentaje de radiación interceptada, que se correlacionan con la producción de forraje (Gobbi et al. 2009). Sin embargo, en trébol pata de pájaro no se ha generado suficiente conocimiento en México, sobre su productividad y dinámica de crecimiento (Álvarez et al. 2020a). Por lo anterior, el objetivo del presente trabajo fue determinar el efecto del momento del corte sobre la respuesta productiva de tres genotipos de Lotus corniculatus L.

\section{MATERIALES Y MÉTODOS}

\section{Área de estudio}

El estudio se llevó a cabo del 4 de mayo al 22 de junio del 2019, en condiciones de invernadero en la Universidad Autónoma Agraría Antonio Narro, Unidad Saltillo ( $25^{\circ} 23^{\prime} 12.7^{\prime \prime}$ LN y $101^{\circ} 00^{\prime}$ 9.8" LO a $1783 \mathrm{msnm}$ ). El clima es semiseco, con temperaturas superiores a los $18{ }^{\circ} \mathrm{C}$ y menores a los cero ${ }^{\circ} \mathrm{C}$. La precipitación media anual es de 340 $\mathrm{mm}$ (Climate-Data-org 2010). Las temperaturas interiores del invernadero se registraron mediante un higrómetro digital modelo WS08, las cuales variaron entre 26.8 a $32.3^{\circ} \mathrm{C}$, las medias entre 22.0 y $25.7^{\circ} \mathrm{C}$, y las minimas entre 15.5 y $19.1^{\circ} \mathrm{C}$ (Figura 1).

\section{Metodología}

Se utilizaron los genotipos 226796, 255301 y 232098, reproducidos en macetas de $17 \times 17 \mathrm{~cm}$, calibre 400 de $1 \mathrm{~kg}$ de capacidad. Se transplantaron el 8 de febrero de 2019 a un sustrato de tierra de monte + arena de rio (1:1), con textura arena-migajón, con una densidad aparente de $1.13 \mathrm{~g} \mathrm{~cm}^{3}$, materia orgánica del $4.53 \%$, carbono orgánico de $2.63 \%$ y nitrógeno total de $6.67 \%$ (Laboratorio de suelos de la UAAAN-Saltillo). Se aplicaron riegos a capacidad de campo cada tercer día y a los 84 días se dio un corte de uniformización a siete $\mathrm{cm}$ de altura residual; posteriormente se efectuaron cortes semanales. La unidad experimental constó de una planta individual, por genotipo, repetición y semana de rebrote.

\section{Variables registradas}

Para estimar composición morfológica se cosechó la biomasa presente a $7 \mathrm{~cm}$ de altura residual en cada unidad experimental, la cual fue separada en hoja, tallo, y material muerto y cada componente se sometió a un proceso de secado a $55^{\circ} \mathrm{C}$ en una estufa Felisa, Modelo FE-243 durante $72 \mathrm{~h}$ a peso constante. El rendimiento de forraje fue la suma de los componentes morfológicos ( $\mathrm{g}$ MS planta $^{-1}$ ). Para la relación hoja:tallo se tomo, de la composición morfológica, el peso de hoja y se dividió entre el del tallo. Para la altura de planta se tomaron 10 lecturas por repetición con una regla de madera de $100 \mathrm{~cm}$, la altura de la planta fue la distancia de la parte basal hasta el último componente morfológico superior. Se colectaron diez tallos al azar por repetición a los cuales se les separó la hoja y se sometieron a un secado por 72 horas a $55^{\circ} \mathrm{C}$ a peso constante, y se obtuvo el promedio del peso por tallo individual (g tallo $\left.{ }^{-1}\right)$.

\section{Análisis estadístico}

Los datos fueron analizados usando un diseño completamente al azar, con tres repeticiones. Para determinar el efecto de genotipo y edad de rebrote, se 


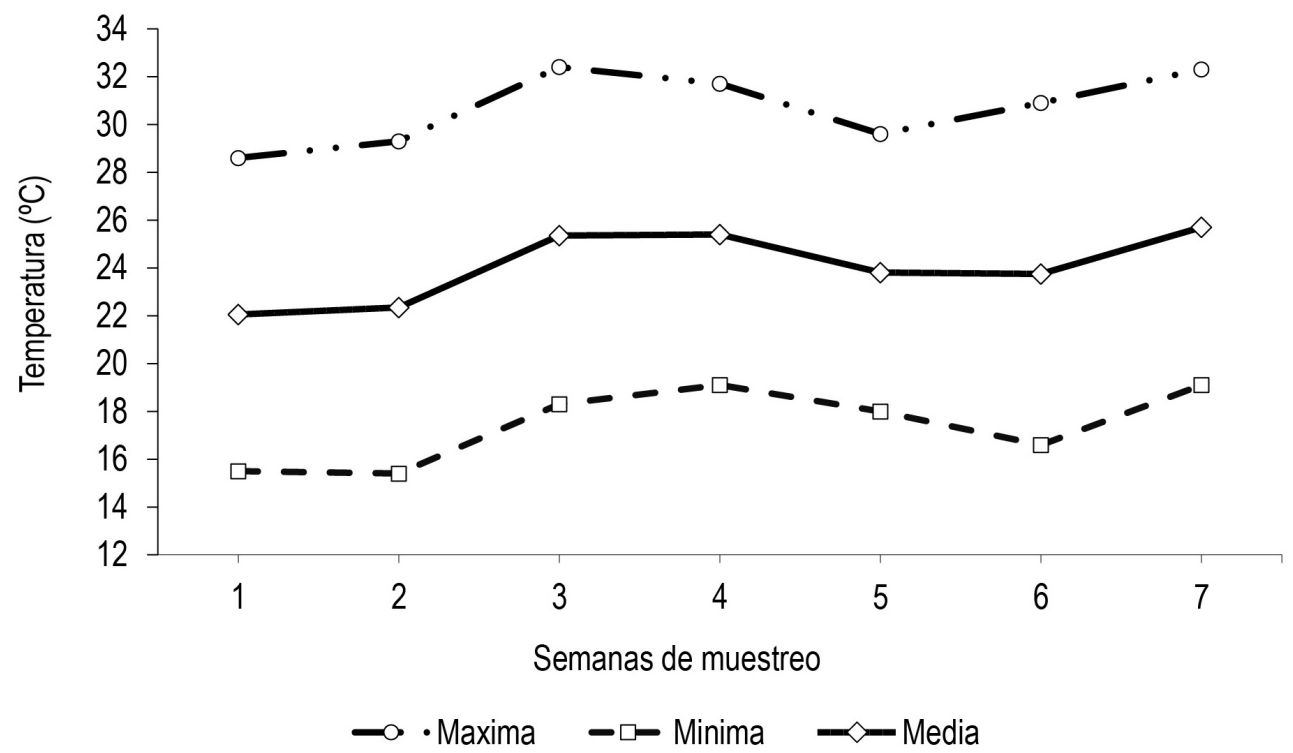

Figura 1. Temperaturas máximas, medias y mínimas interiores, durante el periodo de estudio del 4 de mayo 36 al 22 de junio del 2019. Saltillo, Coahuila, México.

realizó un análisis de varianza con el procedimiento PROC GLM del SAS para Windows versión 9.0 y se realizó una comparación de medias con la prueba Tukey $(\alpha=0.05)$.

\section{RESULTADOS Y DISCUSIÓN}

\section{Rendimiento de forraje}

En la Tabla 1, se muestran la producción de materia seca de tres genotipos de L. corniculatus, cosechados a diferentes días de rebrote (DDR). En los promedios, en el genotipo 255301 y en el 232098 , los mayores valores se registraron de los 35 a los 49 DDR. El el genotipo 226796 el mayor rendmiento se presentó a los 49 DDR. En contraste, los más bajos resultados se presentaron a los 7 DDR $(p<0.05)$. Entre genotipos no se presentaron diferencias estadísticas $(p>0.05)$, a excepción de la primera y tercera semana de rebrote, donde el 232098 sobresalió respecto al 226796 y 255301 ( $p<0.05)$. Por tanto, la producción de forraje, se vio afectado positivamente por la edad de rebrote; a mayores DDR mayor fue el rendimiento de forraje, lo cual puede ser reflejo de la acumulación de tallos a nivel del área basal y el momento del corte (Da Silva et al. 2010, Scheffer et al. 2011). Al respecto, Álvarez et al. (2020b) encontraron un $27 \%$ menos rendimiento en un corte temprano (27 DDR), respecto a un corte tardío (63 DDR). Así mismo, Álvarez et al. (2018), reportaron que genotipos como 202700 de L. corniculatus, no responden favorablemente a un corte temprano de 33 DDR, ya que, disminuyen su rendimiento, área foliar y se incrementa la presencia de malezas.

\section{Composición morfológica}

Se presentaron diferencias entre componentes morfológicos, a través de las edades de rebrotes $(p \leq 0.05)$. Independientemente del genotipo, se registró mayor cantidad de hoja y tallo a los 35,42 y 49 DDR con $3.98,3.84$ y $3.93 \mathrm{~g} \mathrm{MS} \mathrm{planta}^{-1}$ para hoja y $1.46,1.68$ y $1.58 \mathrm{~g} \mathrm{MS}$ planta $^{-1}$ para el tallo, respectivamente. En el material muerto la mayor cantidad se registró a los 42 y 49 DDR, con 0.12 y 0.13 g MS planta $^{-1}$, respectivamente $(p<0.05)$. Los menores valores de todos los componentes morfológicos los encontramos a los 7 DDR, para hoja $0.32 \mathrm{~g} \mathrm{MS} \mathrm{planta}^{-1}$, para tallo $0.06 \mathrm{~g} \mathrm{MS} \mathrm{planta}^{-1} \mathrm{y}$ nulo para material muerto (Figura 2). No obstante, en el porcentaje de aportación al rendimiento de forraje fue diferente a la aportación en g MS planta ${ }^{-1}$. Independientemente del genotipo, la hoja tuvo mayor 
Tabla 1. Rendimiento de material seca ( $\mathrm{g} \mathrm{MS} \mathrm{planta}{ }^{-1}$ ) de tres genotipos de L. corniculatus, cosechados a diferente edad de rebrote.

\begin{tabular}{|c|c|c|c|c|c|}
\hline \multirow{2}{*}{$\begin{array}{c}\text { Días de rebrote } \\
\text { (DDR) }\end{array}$} & \multicolumn{3}{|c|}{ Genotipos } & \multirow{2}{*}{$\dot{X}$} & \multirow{2}{*}{ EEM } \\
\hline & 226796 & 255301 & 232098 & & \\
\hline 7 & $0.33^{E b}$ & $0.24^{D b}$ & $0.60^{D a}$ & $0.72^{E}$ & 0.033 \\
\hline 14 & $1.58^{D a}$ & $1.07^{C D a}$ & $1.37^{D a}$ & $1.62^{D}$ & 0.238 \\
\hline 21 & $1.22^{D b}$ & $1.62^{C b}$ & $2.47^{\mathrm{Ca}}$ & $2.39^{B}$ & 0.157 \\
\hline 28 & $3.36^{C a}$ & $3.15^{B a}$ & $3.50^{B a}$ & $4.17^{B}$ & 0.569 \\
\hline 35 & $5.15^{B a}$ & $5.69^{A a}$ & $5.16^{A a}$ & $5.48^{A}$ & 0.323 \\
\hline 42 & $5.55^{A B a}$ & $5.95^{A a}$ & $5.46^{A a}$ & $5.57^{A}$ & 0.408 \\
\hline 49 & $6.02^{A a}$ & $5.28^{A a}$ & $5.62^{A a}$ & $4.73^{A}$ & 0.274 \\
\hline$\dot{X}$ & $3.32^{a}$ & $3.28^{a}$ & $3.45^{a}$ & 3.35 & 0.116 \\
\hline EEM & 0.179 & 0.389 & 0.279 & 0.121 & \\
\hline
\end{tabular}

aportación en los primeros 7 DDR y menor a los 42 y 49 DDR, con un 86 y $68 \%$, respectivamente. En contraste, el tallo y material muerto, se incrementó conforme avanzó la edad de la planta, ya que a los 7 DDR fue de 14 y $0 \%$, y finalizó a los 49 DDR con un 28 y $2 \%$, para el tallo y material muerto, respectivamente. Sobre lo mismo Álvarez et al. (2020b), reportaron que la hoja representa hasta un $55 \%$ del rendimiento, seguida por el tallo $(30.5 \%)$, material muerto $(8.5 \%)$ y maleza $(4.5 \%)$, registrando una mayor cantidad de cada componente al cosecharse a los 62 DDR y menor a los 33 DDR. En contraste, una disminución en el aporte de la hoja al rendimiento, conforme avanza la edad de la planta, puede ser el resultado de una compensación en el incremento de la biomasa de los tallos, por un mayor periodo de crecimiento, que conlleva a un mayor tiempo produciendo fotosintatos (Pereira et al. 2017). A medida que la planta aumenta la edad de rebrote, esta invierte más recursos en la elongación de tallos en un intento de colocar masa foliar en la parte superior del dosel (Da Silva y Hernandéz 2010). También, la presencia de material muerto a una mayor edad de la planta, puede ser consecuencia de un autosombreado a nivel del área basal, cuando se presentan condiciones favorables de crecimiento (Parsons et al. 2011).

\section{Relación hoja:tallo}

La relación hoja:tallo de tres genotipos de Lotus corniculatus L. se presenta en la Tabla 2. Independiente del genotipo, la mayor relación hoja respecto al tallo se encontró a los 7 DDR y descendió hasta los 35,42 y $49 \mathrm{DDR}$, por lo que en el promedio y en el genotipo 226796 la diferencia fue altamente significativa $(p<.0001)$. La mayor relación hoja:tallo promedio entre genotipos se registró en el 255301, respecto al menor 232098 ( $p \geq 0.05$ ). Únicamente en los días 21, 28 y 49 DDR no se presentaron diferencias estadísticas entre genotipos $(p \geq 0.05)$. Los resultados concuerdan con lo reportado por Álvarez et al. (2020b), quienes reportaron una mayor relación hoja:tallo a los 33 DDR y menor a los 62 DDR, con valores entre 2.8 a 1.8. Así mismo, cortes más frecuentes, en una etapa de crecimiento acelerado con mayor cantidad de hojas jóvenes respecto al tallo, donde el crecimiento de las plantas es menor, es debido a una mayor densidad de tallos, pero de menor peso individual (Barbosa et al. 2011) y en contraste, una menor relación hoja:tallo a mayor edad de la planta, es el resultado de una reducción del número de tallo por planta, pero de mayor peso individual (Giacomini et al. 2009).

\section{Altura de planta}

En la Tabla 3, se presentan los cambios en la altura de la planta de genotipos de L. corniculatus, cosechados a diferentes edades de la planta $(p<$ $0.05)$. En el promedio las menores alturas se registraron a los 7, 14 y 21 DDR, aunque en los genotipos 226796 y 232098 se registaron a los 7 y 14 DDR y en el 255301 a los 7 DDR. Al final del estudio, se presentaron las mayores alturas; en el promedio y 


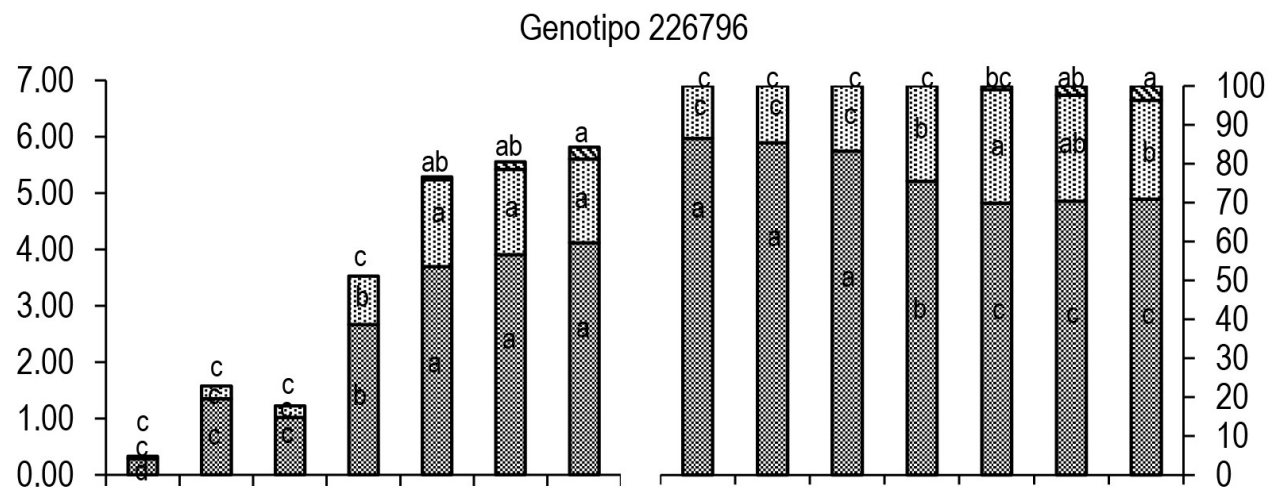

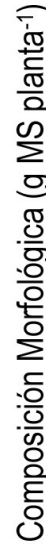

Genotipo 255301
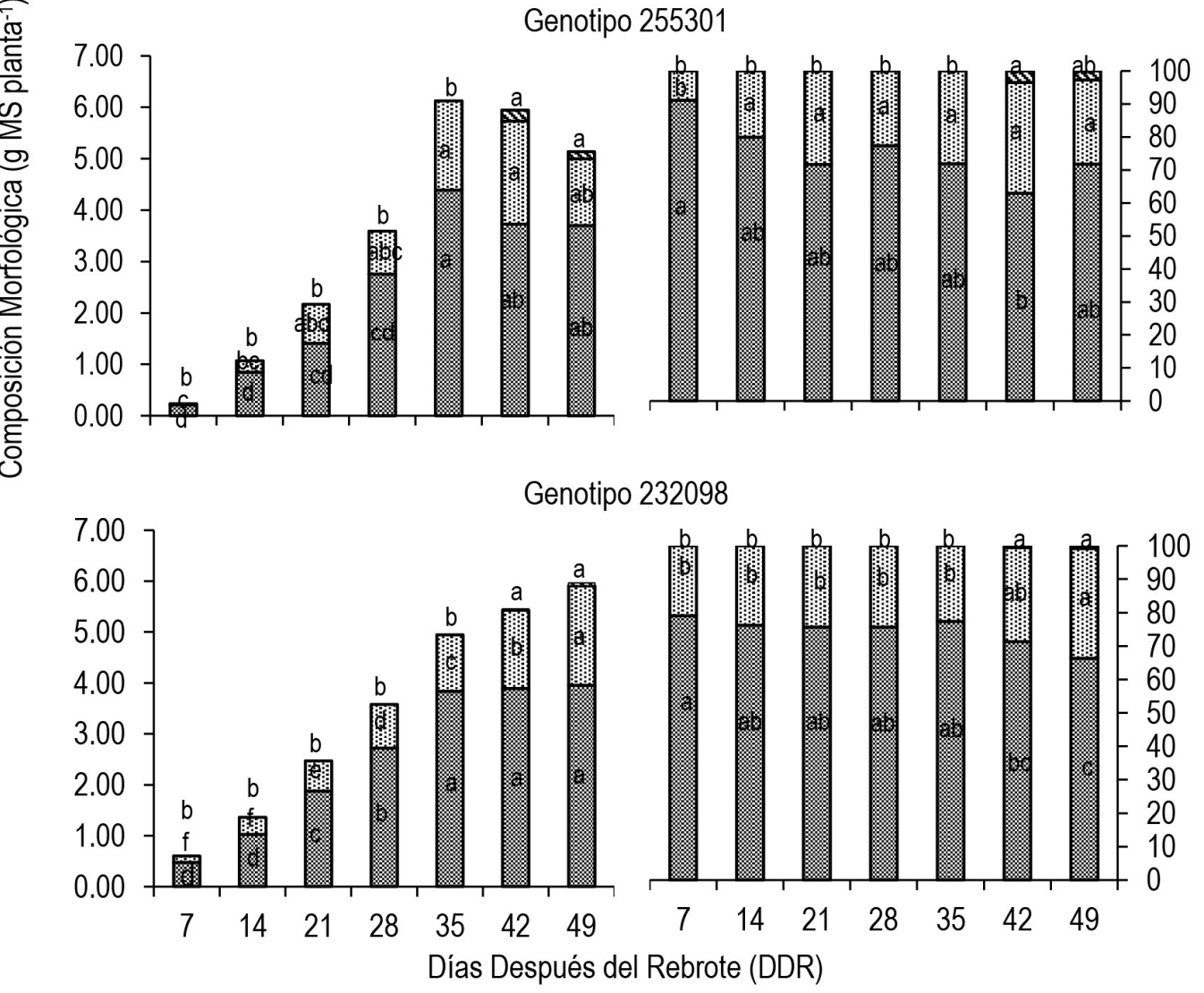

Hoja Tallo 霏 $\quad$ Material muerto $\mathbb{8}$

Figura 2. Cambios en la composición morfológica de tres genotipos de trébol pata de pájaro ( $L$. corniculatus) cosechados a diferente edad de rebrote.

los genotipos 232098 y 255301, con 33.7, 33.5 y $38.7 \mathrm{~cm}$, respectivamente. En el 226796 los mayores fueron a los 35,42 y 49 DDR ( $p>0.05)$. La comparación entre genotipos, mostró los mayores valores en el genotipo 255301 respecto a los genotipos 226796 y al 232098. A excepción de los 7 y 28 DDR, el resto de las edades presentaron diferencias significativas $(p>0.05)$. La altura de planta se incrementó a una mayor edad de la planta, lo que concuerda con un mayor intervalo de corte de $70 \mathrm{DDR}$, respecto a un 
Tabla 2. Relación hoja:tallo (R:H/T) de tres genotipos de L. corniculatus, cosechados a diferente edad de rebrote.

\begin{tabular}{|c|c|c|c|c|c|}
\hline \multirow{2}{*}{$\begin{array}{c}\text { Días de rebrote } \\
\text { (DDR) }\end{array}$} & \multicolumn{3}{|c|}{ Genotipos } & \multirow{2}{*}{$\dot{X}$} & \multirow{2}{*}{ EEM } \\
\hline & 226796 & 255301 & 232098 & & \\
\hline 7 & $6.44^{\text {Aab }}$ & $10.86^{A a}$ & $3.90^{A b}$ & $5.71^{A}$ & 2.276 \\
\hline 14 & $5.86^{A B a}$ & $4.00^{B a b}$ & $3.34^{A B b}$ & $4.06^{B}$ & 0.674 \\
\hline 21 & $5.06^{B a}$ & $3.91^{B a}$ & $3.17^{A B a}$ & $3.76^{B}$ & 1.803 \\
\hline 28 & $3.10^{C a}$ & $3.52^{B a}$ & $3.16^{A B a}$ & $3.16^{B C}$ & 0.449 \\
\hline 35 & $2.40^{C b}$ & $2.56^{B b}$ & $3.44^{A B a}$ & $2.57^{C}$ & 0.135 \\
\hline 42 & $2.59^{\mathrm{Ca}}$ & $1.90^{B b}$ & $2.54^{A B a}$ & $2.39^{C}$ & 0.163 \\
\hline 49 & $2.78^{C a}$ & $2.88^{B a}$ & $2.03^{B a}$ & $3.00^{C}$ & 0.476 \\
\hline$\dot{X}$ & $4.03^{a b}$ & $4.23^{a}$ & $3.08^{b}$ & 3.78 & 0.397 \\
\hline EEM & 0.461 & 1.686 & 0.500 & 0.426 & \\
\hline
\end{tabular}

Valores con la misma literal mayúscula en cada columna y la misma literal minúscula en cada fila no son diferentes (Tukey; $\alpha=0.05$ ); EEM = error estándar de la media. $\dot{X}=$ Media de genotipos y DDR.

Tabla 3. Altura de planta $(\mathrm{cm})$ de tres genotipos de L. corniculatus, cosechados a diferente edad de rebrote.

\begin{tabular}{|c|c|c|c|c|c|}
\hline \multirow{2}{*}{$\begin{array}{l}\text { Días de rebrote } \\
\text { (DDR) }\end{array}$} & \multicolumn{3}{|c|}{ Genotipos } & \multirow{2}{*}{$\dot{X}$} & \multirow{2}{*}{ EEM } \\
\hline & 226796 & 255301 & 232098 & & \\
\hline 7 & $12.0^{\mathrm{Ca}}$ & $11.8^{D a}$ & $11.7^{D a}$ & $12.8^{C}$ & 1.354 \\
\hline 14 & $14.0^{C a b}$ & $15.5^{C D a}$ & $13.3^{D b}$ & $16.0^{C}$ & 0.577 \\
\hline 21 & $14.8^{B C b}$ & $19.5^{\mathrm{Ca}}$ & $17.5^{\mathrm{Ca}}$ & $19.6^{C}$ & 0.781 \\
\hline 28 & $24.0^{A B a}$ & $27.3^{B a}$ & $18.8^{C a}$ & $24.3^{B}$ & 4.116 \\
\hline 35 & $30.0^{A a}$ & $31.5^{A B a}$ & $20.0^{B C b}$ & $27.5^{B}$ & 0.577 \\
\hline 42 & $29.5^{A a}$ & $33.5^{A B a}$ & $22.5^{B b}$ & $28.5^{A B}$ & 2.000 \\
\hline 49 & $29.0^{A b}$ & $38.7^{A a}$ & $33.5^{A a b}$ & $33.7^{A}$ & 2.108 \\
\hline$\dot{X}$ & $21.9^{b}$ & $25.4^{a}$ & $19.6^{b}$ & 22.3 & 1.201 \\
\hline EEM & 3.262 & 2.561 & 1.072 & 1.923 & \\
\hline
\end{tabular}

Valores con la misma literal mayúscula en cada columna y la misma literal minúscula en cada fila no son diferentes (Tukey, $\alpha=0.05$ ); EEM = error estándar de la media. $\dot{X}=$ Media de genotipos y DDR.

menor de 33 DDR, relacionado con cortes más frecuentes donde se obtienen alturas de planta más bajas (Álvarez et al. 2020a, Scheffer et al. 2011). También las mayores y menores alturas se relaciona con las condiciones de clima bajo las cuales se desarrollen las plantas, como fotoperiodo, temperatura y humedad (Parsons et al. 2011), ya que, en condiciones favorables las plantas aceleran su crecimiento y presentan cambios en la altura y rendimiento, por lo que la altura de una pradera da idea del forraje producido (Rojas et al., 2016). De acuerdo con García et al. (2014) registraron las mayores alturas de planta en un rango de temperaturas minimas de $14{ }^{\circ} \mathrm{C}$ y máximas de $30{ }^{\circ} \mathrm{C}$, mostrando que el crecimiento de la especie Lotus corniculatus L., disminuye con el descenso de la temperatura.

\section{Peso de tallo}

Los cambios en el peso por tallo individual de tres genotipos de trébol pata de pájaro, se muestran en la Tabla 4. Los mayores pesos promedios se registraron a partir de los 28 DDR y los menores en los 7,14 y 21 DDR ( $p \geq 0.05$ ). No obstante, en el genotipo 226796, fue a partir de los 35 DDR, en el 255301 a partir de los 42 DDR y el 232098 a los 49 DDR $(p>0.05)$. Para los tres genotipos el menor valor se registró a los 7 DDR. La comparación de los promedios de los genotipos muestran que el 255301 superó al resto con $0.07 \mathrm{~g}$ tallo $^{-1}$ ( $\mathrm{p}<$ $0.05)$, y únicamente fueron similares los tres genotipos a los 14 DDR $(p>0.05)$. Las plantas se encuentran en fases finales de crecimiento, el componente más importante del rendimiento de materia seca es el peso del tallo (Hernández et al. 2000), por lo que el rendimiento se mantiene en una misma tendencia positiva similar al peso de los tallos (Tabla 1 y Figura 2). Conforme avanza la edad de la planta, hay una competencia intraespecífica por luz, nutrientes y es- 
Tabla 4. Peso de tallo ( $\mathrm{g}_{\text {tallo }}{ }^{-1}$ ) de tres genotipos de L. corniculatus, cosechados a diferente edad de rebrote.

\begin{tabular}{|c|c|c|c|c|c|}
\hline \multirow{2}{*}{$\begin{array}{l}\text { Días de rebrote } \\
\text { (DDR) }\end{array}$} & \multicolumn{3}{|c|}{ Genotipos } & \multirow{2}{*}{$\dot{X}$} & \multirow{2}{*}{ EEM } \\
\hline & 226796 & 255301 & 232098 & & \\
\hline 7 & $0.02^{C a b}$ & $0.02^{E a}$ & $0.01^{D b}$ & $0.02^{B}$ & 0.003 \\
\hline 14 & $0.03^{C b a}$ & $0.03^{D E a}$ & $0.02^{C D a}$ & $0.03^{B}$ & 0.003 \\
\hline 21 & $0.03^{C b b}$ & $0.05^{C D a}$ & $0.03^{B C b}$ & $0.04^{B}$ & 0.005 \\
\hline 28 & $0.05^{B a b}$ & $0.06^{\mathrm{Ca}}$ & $0.04^{A B C b}$ & $0.06^{A}$ & 0.006 \\
\hline 35 & $0.08^{A a}$ & $0.08^{B C a}$ & $0.05^{A B b}$ & $0.07^{A}$ & 0.010 \\
\hline 42 & $0.08^{A b}$ & $0.10^{A B a}$ & $0.05^{A B c}$ & $0.08^{A}$ & 0.006 \\
\hline 49 & $0.08^{A b}$ & $0.12^{A a}$ & $0.06^{A b}$ & $0.07^{A}$ & 0.009 \\
\hline$\dot{X}$ & $0.05^{b}$ & $0.07^{a}$ & $0.04^{c}$ & 0.05 & 0.001 \\
\hline EEM & 0.007 & 0.011 & 0.008 & 0.021 & \\
\hline
\end{tabular}

Valores con la misma literal mayúscula en cada columna y la misma literal minúscula en cada fila no son diferentes (Tukey, $\alpha=0.05$ ); EEM $=$ error estándar de la media.

pacio, lo que ocasiona que a edades tempranas, los tallos tiene menor peso, pero hay mayor cantidad de estos, caso contrario a una edad madura de los macollos, con menos tallos pero de mayor peso (Castro et al. 2013).

El comportamiento productivo de los genotipos de Lotus corniculatus fue afectada por la edad de cosecha. Mientras que el rendimiento de forraje, al- tura de la planta y peso por tallo individual, incrementaron con el avance de edad de la planta. El genotipo 255301 tuvo la mayor altura y relación hoja:tallo. La relación hoja:tallo fue mayor a menor edad de la planta, mientras que la hoja fue el componente morfológico que más aportó al rendimiento, aunque se redujo al incrementarse la edad de rebrote, mientras el tallo y el material muerto la incrementaron.

\section{LITERATURA CITADA}

Álvarez AV, Mendoza PSI, García DSG, Guerrero RJD, Rojas GAR, Encina DJA (2020a) Respuesta productiva de trébol pata de pájaro en función del porcentaje de luz interceptada. Revista Mexicana de Ciencias Agrícolas Pub. Esp. Núm. 24: 105-116.

Álvarez AV, Guerrero RJD, García DSG, Ortega CME, Mendoza PSI, Joaquín CS (2020b) Acumulación de forraje de Lotus corniculatus L., en función a diferentes estrategias de cosecha. Revista Mexicana de Ciencias Pecuarias 11: 1087-1100.

Álvarez VP, García DSG, Guerrero RJD, Mendoza PSI, Ortega CME, Hernández GA (2018) Comportamiento productivo de Lotus corniculatus L. dependiente de la estrategia de cosecha. Revista Agrociencia 52: 1081-1093.

Barbosa RA, Nacimiento-Jr D, Vilela HH, Da-Silva CS, Batista-Euclides PV, Sbrissia FA, Da-Lana SB (2011) Morphogenic and structural characteristics of guinea grass pastures submitted to three frequencies and two defoliation severities. Revista Brasileira de Zootecnia 40: 947-954.

Castro RR, Hernández-Garay A, Ramírez RO, Aguilar BG, Enríquez QJF, Mendoza PSI (2013) Crecimiento en longitud foliar y dinámica de población de tallos de cinco asociaciones de gramíneas y leguminosa bajo pastoreo. Revista Mexicana de Ciencias Pecuarias 4: 201-215.

Climate-Data-org (2010) Clima Saltillo. https://es.climate-data.org/america-del-norte/mexico/coahuila-de-zaragoza/saltillo-4988/. Fecha de consulta: 20 septiembre de 2020.

Da Silva SC, Hernández GA (2010) Manejo del pastoreo en praderas tropicales. In: Velazco ZME (ed.) Los forrajes y su impacto en el trópico. 1ra. ed. Universidad Autónoma de Chiapas. México. pp: 63-95. 
Da Silva NM, De Souza LI, De Moura ZA, Gonçalves DAA, Ferreira DJJ, Buranelo TDL, Araujo PRM (2020) Morphogenetic and structure characteristics of marandu grass subjected to grazing management strategies. Biological Rhythm Research 51: 898-906.

Escaray FJ, Menendez AB, Garriz A, Pieckenstain FL, Estrella MJ, Castagno LN, Carrasco P, Sanjuan J, Ruiz OA (2012) Ecological and agronomic importance of the plant genus Lotus. Its application in grassland sustainability and the amelioration of constrained and contaminated Soils Plant Science 182: 121-133.

García BDV, Guerrero RJD, García DSG, Lagunes RSA (2014) Rendimiento y calidad de forraje de genotipos de Lotus corniculatus L., en el Estado de México. Nova Scientia 7: 170-189.

Giacomini AA, Da-Silva CS, Sarmento DLDO, Zeferino VC, Souza JS, Da-Trindade KJ, Guarda VDelA, Junior ND (2009) Growth of marandu palisadegrass subjected to strategies of intermittent stocking. Sci Agric Piracicaba 66: 733-741

Gobbi KF, Garcia R, Garcez NAF, Pereira OG, Ventrella MC, Rocha GC (2009) Morphological and structural characteristics and productivity of Brachiaria grass and forage peanut submitted to shading. Revista Brasileira Zootecnia 38: 1645-1654.

Hernández GA, Matthew C, Hodgson J (2000) The influence of defoliation height on dry-matter partitioning and CO2 exchange of perennial ryegrass miniature swards. Grass Forage Science 55: 372-376.

Naydenova G, Vasileva V, Mitev D (2015) Productivity of Bulgarian pasture ecotypes perennial legumes. Journal of Mountain Agriculture on the Balkans 18: 972-982.

Parsons A, Rowarth J, Thornley J, Newton P (2011) Primary production of grasslands, herbage accumulation and use, and impacts of climate change. In: Lemaire G, Hodgson J, Chabbi A (eds). Grassland productivity and ecosystem services. CAB International Editors. Paris, France. pp: 3-18.

Pereira JC, Gomes FK, Oliveira DBLM, Lara ASM, Bernardes TF, Casagrande DR (2017) Defoliation management affects morphogenetic and structural characteristics of mixed pastures of brachiaria grass and forage peanut. African Journal of Range and Forage Science 34: 13-19.

Rojas GAR, Hernández GA, Cansino SJ, Maldonado PMDLÁ, Mendoza PSI, Álvarez VP, Joaquín Torres BM (2016) Comportamiento productivo de cinco variedades de alfalfa. Revista Mexicana de Ciencias Agrícolas 7: 1855-1866.

Scheffer BMS, Brustolin R, Dall AM (2011) Performance of Lotus corniculatus L. genotypes submitted to cutting interval: subsidies to a breeding program. Revista Brasileira de Zootecnia 40: 1645-1650.

Sousa BML, Do Nascimento JrD, Da Silva SC, Monteiro HCDF, Rodrigues CS, Da Fonseca DM, Da Silveira MCT, Sbrissia AF (2010) Morphogenetic and structural characteristics of Andropogon grass submitted to different cutting heights. Revista Brasileira de Zootecnia 39: 2141-2147.

Vasileva V (2014) Productivity of dry aboveground and root mass in mixtures. Journal of Mountain Agriculture on the Balkans 17: 956-969. 